\title{
Estimating the Impact of Fingerprint Image Enhancement Algorithms for Better Minutia Detection
}

\author{
M.Rajinikannan \\ Department of MCA \\ PSNA CET, \\ Dindigul. -624622
}

\author{
D.Ashok Kumar \\ Department of Computer Science \\ VSS Government Arts College, \\ Pulankurichi - 630413
}

\author{
R.Muthuraj \\ Department of Mathematics \\ PSNA CET, \\ Dindigul. -624622
}

\begin{abstract}
A very important step in automatic fingerprint recognition system is to automatically and reliably extract minutia from the input fingerprint images. But the performance of a minutia extraction algorithm relies heavily on the quality of the input fingerprint images and the image processing techniques used for enhancing the ridges and valleys of the fingerprint image and make the minutia distinguishable from the other parts of the fingerprint image. In this paper, we have evaluated three models of minutia detection systems which are particularly very much different in the way of enhancing the input image for minutia detection. It is proposed to use FFT and Gabor Filter based on frequency and orientation image enhancement technique instead of directional convolution and grey scale. Finally we have compared the performance of image enhancement algorithms using the number of minutia in all the three cases with the values of a human observer.
\end{abstract}

\section{Categories and Subject Descriptors}

G.4 - MATLAB is a high-level technical computing language and interactive environment for algorithm development, data visualization, data analysis, and numeric computation. Using the MATLAB product, you can solve technical computing problems faster than with traditional programming languages, such as $\mathrm{C}$, $\mathrm{C}++$, and FORTRAN.

\section{Keywords}

Fingerprint Image Enhancement, Ridge Endings, Ridge Bifurcation, Histogram, FFT, Gabor Filter, Minutia Detection.

\section{INTRODUCTION}

Fingerprint recognition is part of the larger field of Biometrics. Other techniques of biometrics include face recognition, voice recognition, hand geometry, retinal scan, ear surface and so on.

\subsection{The Finger Print Image and Minutia}

A fingerprint is the feature pattern of one finger. Strong evidences shows that each fingerprint is unique [3]. Each person has his own fingerprints with the permanent uniqueness. So fingerprints have been used for identification and forensic investigation for a long time [3].

A fingerprint is the pattern of ridges and valleys on the finger tip. A fingerprint is thus defined by the uniqueness of the local ridge characteristics and their relationships. Minutia points are these local ridge characteristics that occur either at a ridge ending or a ridge bifurcation. A ridge ending is defined as the point where the ridge ends abruptly and the ridge bifurcation is the point where the ridge splits into two or more branches.

A fingerprint expert is often able to correctly identify the minutia by using various visual clues such as local ridge orientation, ridge continuity, ridge tendency, etc, as long as the ridge and furrow structures are not corrupted completely [10]. It is possible to develop an enhancement algorithm that exploits these visual clues to improve the clarity of ridge structures in corrupted fingerprint images.

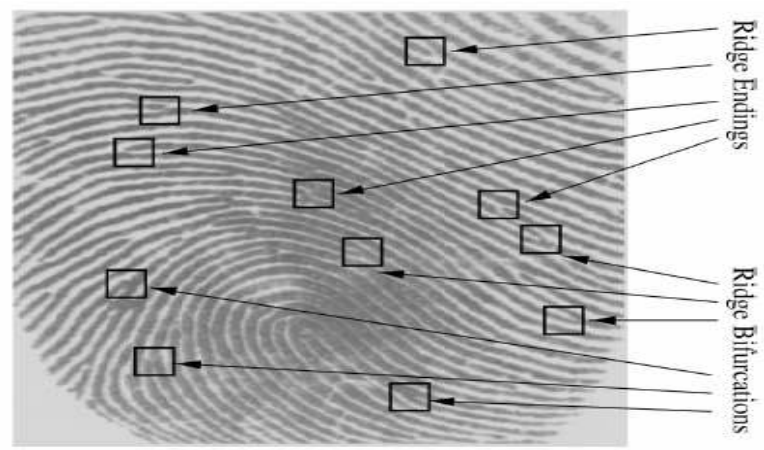

Figure 1. The Ridge Endings and Bifurcation.

The following diagram shows the clear view of a minutia. Minutia is characterized by its location and orientation.

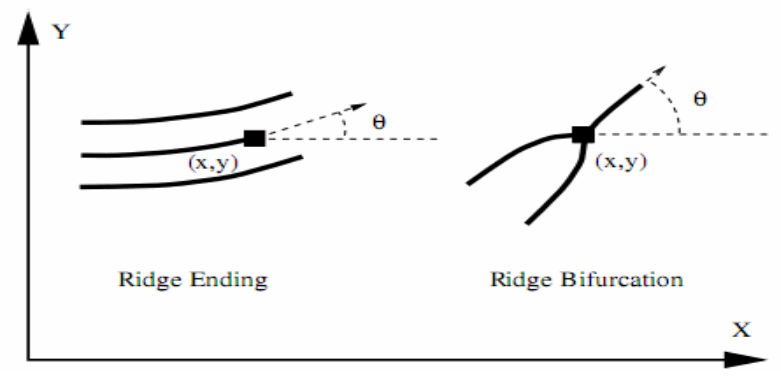

Figure 2. The Characteristic Attributes of a Minutia. 


\subsection{Importance of Fingerprint Image Enhancement Algorithms}

There are several steps in a typical fingerprint recognition system, and there are several types of algorithms in each of these stages, we can broadly reduce these categories of algorithms as follows:

$>$ Fingerprint Image Enhancement (Ridge and Valley Enhancement) Algorithms

$>$ Minutia Detection Algorithms

$>$ Minutia Matching Algorithms

Fingerprint Image Enhancement (Ridge and Valley Enhancement) Algorithms are the most important step in any fingerprint based recognition systems since the results of Minutia Detection Algorithms and the Minutia Matching Algorithms very much depend on the clarity of the enhanced fingerprint image.

\subsection{Previous works}

The quality of the ridge features in a fingerprint image is important, since the ridges form the basis for minutia extraction. In a perfect image, the ridges and valleys should not only alternate but also flow without any perturbations. However, in real world, a fingerprint image may not always be perfect due to noise which distorts the ridge structures. This corruption may occur as a result of impression conditions such as scars or a non-uniform contact with the fingerprint scanner [8]. It is here, image enhancement techniques are often employed to reduce the noise and enhance the definition of ridges against valleys.

There has been lot of interesting work done in enhancing fingerprints. Sherlock [7] proposed enhancing the features in a fingerprint image by directional Fourier filtering. This frequency domain filtering is computationally less expensive than the spatial convolution of the image with filters. The filtered image is usually binarized or thinned for feature extraction. But there has been an effort to extract features from gray scale images, Maio and Maltoni [2] proposed an algorithm to extract features from gray scale images. The feature extraction algorithm has usually been employed on thinned images. Jain [1] and Ratha [6]developed algorithms for thinned images, their approach has involved local neighborhood based processing on the images.

Many authors have identified the need to perform post processing on fingerprint images to remove the false minutia, Ratha et al. [6], where the minutia are validated based upon heuristics like distance. Since the fingerprint based system rely on matching between the query fingerprint and the database fingerprint, classification of the database results in the query only searching in a particular class. Many attempts [2][4] have been made to classify the fingerprints based upon core as well as delta points, these have been point based approach. The matching forms the heart of any fingerprint; the query fingerprint of even a client is usually a transformed version of the database fingerprint. This involved registration of the images before obtaining the match. There have been several prior approaches that addressed this. Ranade and Rosenfield [5] proposed an iterative approach for obtaining point correspondences.

The fingerprint enhancement techniques proposed by Jain [6], is based on the convolution of the image with Gabor filters which has the local ridge orientation and ridge frequency. The algorithm includes normalization, ridge orientation estimation, ridge frequency estimation and filtering. Gabor filters are band pass filters that have both frequency-selective and orientation-selective properties [8], thus the ridge are enhanced.

\section{THE MINUTIA DETECTION}

In this paper, we evaluate the performance of three types of image enhancement techniques and their impact in minutia detection. They are

a) The Histogram Based Image Enhancement

b) The FFT Filter Based Fingerprint Image Enhancement

c) The Gabor Filter Based Fingerprint Image Enhancement

The first one "Histogram Based Image Enhancement "is not at all a specific algorithm for finger print image enhancement. It is commonly used to enhance images during all kinds of image processing operations. In other words, we can consider this case as a Minutia Detection without any fingerprint image enhancement.

\subsection{The Image Enhancement Methods under Evaluation}

\subsubsection{The Histogram Based Image Enhancement}

The distribution of gray levels occurring in an image is called gray level histogram. It is a graph showing the frequency of occurrence of each gray level in the image versus the gray level itself. The plot of this function provides a global description of the appearance of the image.

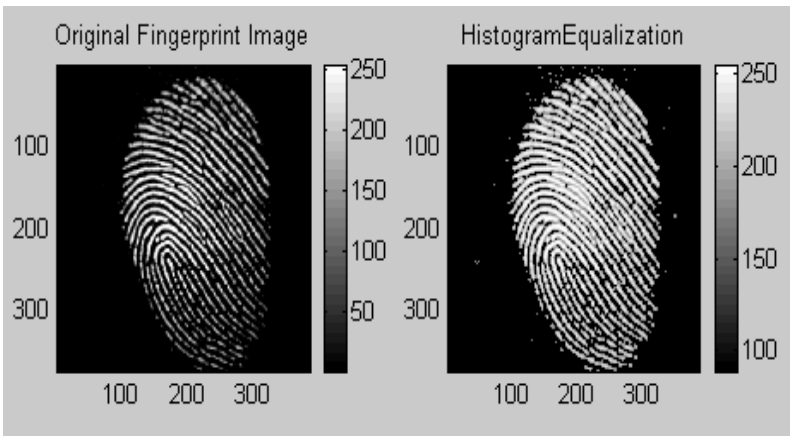

Figure 3. The Histogram Equivalence

Original Image (left) - The Histogram Equivalized (right)

The histogram of a digital image with gray levels in the range $[0$, $\mathrm{L}-1]$ is a discrete function.

$$
P\left(r_{k}\right)=n_{k} / n
$$

Where,

$r_{k}$ is the k'th gray level

$\mathrm{n}_{\mathrm{k}}$ is the number of pixels in the image with that gray level.

$\mathrm{n}$ is the total number of pixels in the image.

$\mathrm{k}=0,1,2, \ldots, \mathrm{L}-1$.

$\mathrm{L}=256$. 
The equation (1), gives an estimate of the probability of occurrence of gray level rk .

\subsubsection{The FFT Filter Based Fingerprint Image Enhancement}

This method [9] has been widely used in earlier works of fingerprint image recognition systems. In this implementation, we divide the image into small processing blocks ( 32 by 32 pixels) and perform the Fourier transform according to:

$$
F(u, v)=\sum_{x=0}^{M-1} \sum_{y=0}^{N-1} f(x, y) \times \exp \left\{-j 2 \pi \times\left(\frac{u x}{M}+\frac{v y}{N}\right)\right\}
$$

For $\mathrm{u}=0,1,2 \ldots 31$ and $\mathrm{v}=0,1,2 \ldots 31$.

In order to enhance a specific block by its dominant frequencies, we multiply the FFT of the block by its magnitude a set of times. Where the magnitude of the original

$$
\mathrm{FFT}=\operatorname{abs}(\mathrm{F}(\mathrm{u}, \mathrm{v}))=|\mathrm{F}(\mathrm{u}, \mathrm{v})| \text {. }
$$

Get the enhanced block according to

$$
g(x, y)=F^{-1} F\left\{(u, v) \times|F(u, v)|^{K}\right\}
$$

Where $\mathrm{F}^{-1}(\mathrm{~F}(\mathrm{u}, \mathrm{v}))$ is done by:

$$
f(x, y)=\frac{1}{M N} \sum_{x=0}^{M-1} \sum_{y=0}^{N-1} F(u, v) \times \exp \left\{j 2 \pi \times\left(\frac{u x}{M}+\frac{v y}{N}\right)\right\}
$$

$$
\text { For } \mathrm{x}=0,1,2 \ldots 31 \text { and } \mathrm{y}=0,1,2 \ldots 31 \text {. }
$$

The $\mathrm{k}$ in equation (3) is an experimentally determined constant, which we choose $\mathrm{k}=0.45$ to calculate. While having a higher " $\mathrm{k}$ " improves the appearance of the ridges, filling up small holes in ridges, having ' $k$ ' value larger can result in false joining of ridges. Thus a termination might become a bifurcation. The following figure presents the image after FFT enhancement.

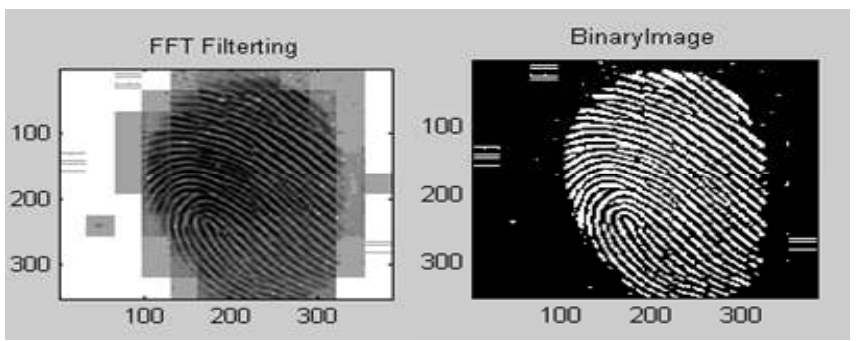

Figure 4. Fingerprint Enhancement by FFT

FFT Filtered Image (left), Enhanced Binary Image (right)

The enhanced image after FFT has the improvements to connect some falsely broken points on ridges and to remove some spurious connections between ridges.

\subsubsection{The Gabor Filter Based Fingerprint Image Enhancement}

The configurations of parallel ridges and furrows with welldefined frequency and orientation in a fingerprint image provide useful information which helps in removing undesired noise. The sinusoidal shaped waves of ridges and furrows vary slowly in a local constant orientation. Therefore, a band-pass filter that is tuned to the corresponding frequency and orientation can efficiently remove the undesired noise and preserve the true ridge and furrow structures. Gabor filters [10] have both frequency selective and orientation selective properties and have optimal joint resolution in both spatial and frequency domains. Therefore, it is appropriate to use Gabor filters as band-pass filters to remove the noise and preserve true ridge/valley structures.

A Gabor filter is used for this process and a suitable value of local variances is taken for carrying out the process of filtering. A Gabor filter takes care of both the frequency components as well as the spatial coordinates. The inputs required to create a Gabor mask are frequency, orientation angle and variances along $\mathrm{x}$ and $\mathrm{y}$ directions. Filtering is done for each block using the local orientation angle and frequency.

Once the ridge orientation and ridge frequency information has been determined, these parameters are used to construct the evensymmetric Gabor filter. A two dimensional Gabor filter consists of a sinusoidal wave of a particular orientation and frequency, modulated by a Gaussian envelope [8]. Gabor filters are employed because they have frequency-selective and orientation-selective properties. These properties allow the filter to be tuned to give the best response to ridges at a specific orientation and frequency in the fingerprint image. Therefore, a properly tuned Gabor filter can be used to effectively preserve the ridge structures while reducing noise. The even-symmetric Gabor filter is the real part of the Gabor function [8].

$$
\begin{aligned}
& G(x, y ; \theta, f)=\exp \left\{-\frac{1}{2}\left[\frac{x_{\theta}^{2}}{\sigma_{x}^{2}}+\frac{y_{\theta}^{2}}{\sigma_{y}^{2}}\right]\right\} \cos \left(2 \pi f x_{\theta}\right), \\
& x_{\theta}=x \cos \theta+y \sin \theta \\
& y_{\theta}=-x \sin \theta+y \cos \theta
\end{aligned}
$$

In equation (5), $\theta$ is the local orientation, $f$ is the frequency, $\sigma_{x}$ and $\sigma_{\mathrm{y}}$ are the standard deviation of the Gaussian envelope.

The finger print image is enhanced by convolving the normalized image with the Gabor filter. The convolution for a pixel requires the pixels corresponding orientation and frequency values, these values are used to construct a Gabor filter specific to that pixel.

Thus the convolution will result in the noise being reduced and the ridges being enhanced, since the Gabor filter only filters along the specified orientation this decreases anything oriented differently. Hence, the filter increases the contrast and reduces the noise. 


\subsection{The other Stages of Processing}

\subsubsection{Binarization}

The feature extraction algorithm in the case of fingerprint's operate on binary images, thus there is a need to convert the grey scale image to a binary image. In which the black pixels represent ridges and the white pixels represent the valleys. Binarization is done to increase the contrast between the ridge and the valley and hence facilitates the extraction of minutia.

The Gabor filter has a zero DC component, which means the resulting filtered image has a mean pixel value of zero. Thus the global threshold for the binarization is zero; if the pixel value is greater than zero its set to one else it is set to zero. The result of binarization is an image which has only values of two pixels representing either a ridge or a valley.

\subsubsection{Thinning}

The binarized image needs to be thinned before the minutia can be extracted from the image. Thinning is a morphological operation that erodes the ridge pixels until they are one pixel wide. The thinning algorithm is one which follows infinite iterations till the ridge is one pixel wide. In each iteration, the neighbourhood of the pixel is considered and a set of pixel-deletion criteria are applied to obtain the skeleton of the image. The algorithm for thinning is as follows

The neighbours of a central pixel are defined in figure 5 .

\begin{tabular}{|c|c|c|}
\hline $\mathrm{P}$ & $\mathrm{P}$ & $\mathrm{P}$ \\
4 & 3 & 2 \\
\hline $\mathrm{P}$ & $\mathrm{P}$ & $\mathrm{P}$ \\
5 & & 1 \\
\hline $\mathrm{P}$ & $\mathrm{P}$ & $\mathrm{P}$ \\
6 & 7 & 8 \\
\hline
\end{tabular}

Figure 5: The neighbours of a pixel

$\Sigma(\mathrm{p} 0)$ is the sum of nonzero neighbours and $\zeta(\mathrm{p} 0)$ is the transition sum of the neighbour of $\mathrm{p}$, which equals the number of transition from 0 to 1 in the ordered set, p1, p2, p3, p4, p5, p6, p7, p8, p1

The thinning rules are as follow:

- $\quad 1<\Sigma(\mathrm{p})<7$

- $\zeta(\mathrm{p})=2$

- $\quad$ pp5p7=0

- $\quad \mathrm{p} 3 \mathrm{p} 5 \mathrm{p} 7=0$

The iterations continue till the binary image becomes skeletonized. The thinning preserves the connectivity of the ridge structure and also forms a skeleton of the fingerprint image. This skeleton image is used for minutia extraction.

\subsubsection{Minutia Extraction}

The next step after enhancement of the image is the extraction of minutia. The enhanced image is binarized first in this step. The skeleton of the image is then formed. The minutia points are then extracted by the following method. The binary image is thinned as a result of which a ridge is only one pixel wide. The minutia points are thus those which have a pixel value of one (ridge ending) as their neighbour or more than two ones (ridge bifurcations) in their neighbourhood. This ends the process of extraction of minutia points.

\subsubsection{Post-Processing}

The minutia points obtained in the above step may contain many spurious minutias. This may occur due to the presence of ridge breaks in the given figure itself which could not be improved even after enhancement. This results in false minutia points which need to be removed. These unwanted minutia points are removed in the post-processing stage. False minutia points will be obtained at the borders as the image ends abruptly. These are deleted using the segmented mask. As a first step, a segmented mask is created. This is created during segmentation carried out in the stage of preprocessing and contains ones in the blocks which have higher variance than the threshold and zeros for the blocks having lower variance. This segmented mask contains all ones in the regions where the image is located and all zeros at the other places.

In this work, we didn't do any post processing to reduce spurious minutia. It counts spurious minutias while estimating the false detection rate.

\section{IMPLEMENTATION RESULTS AND ANALYSIS}

The figure 6 shows the evaluation strategy used in this work.

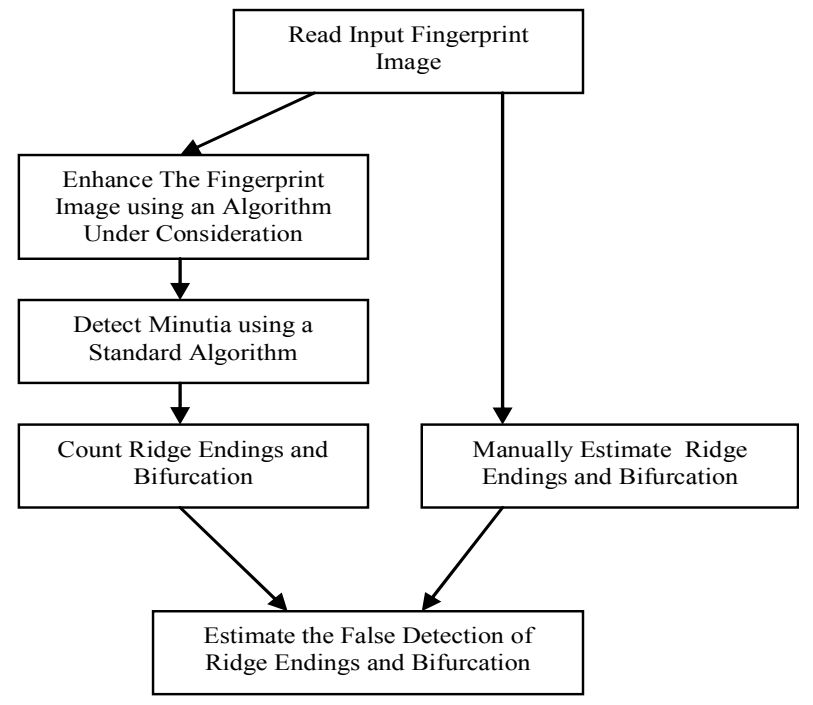

Figure 6: The Evaluation Model

\subsection{Fingerprint Database Used For Evaluation}

A fingerprint database from the FVC2000 (Fingerprint Verification Competition 2000) is used to test the experiment performance. FVC2000 was the First International Competition 
for Fingerprint Verification Algorithms. This initiative is organized by D. Maio, D. Maltoni, R. Cappelli from Biometric Systems Lab (University of Bologna), J. L. Wayman from the U.S. National Biometric Test Center (San Jose State University) and A. K. Jain from the Pattern Recognition and Image Processing Laboratory of Michigan State University.

In FVC2000, four different "sensors" were used to cover the recent advances in fingerprint sensing techniques. In fact, databases 1 and 2 were collected by using two small-size and lowcost sensors (optical and capacitive, respectively). Database 3 was collected by using a higher quality (large area) optical sensor. Finally, images in database 4 were synthetically generated by using this approach described in [7]. Each database was split into a sequestered "test" set of 800 images (set A) and an open "training" set of 80 images made available to participants for algorithm tuning (set B).

Sets of few selected images from the above database were used to evaluate the performance of the algorithms.

\subsection{A Sample Set of Results}

In the following figure presents the impact of three image enhancement approaches in the Detected Ridge Ending and Bifurcation. In the figure, the location marked with "blue stars" are the detected ridge ending and the location marked with "green circles" is the detected bifurcation.

\begin{tabular}{|c|c|c|}
\hline & $\begin{array}{c}\text { The Enhanced and } \\
\text { Thinned Fingerprint Image }\end{array}$ & $\begin{array}{c}\text { The Detected Ridge } \\
\text { Ending and Bifurcation. }\end{array}$ \\
\hline$\Xi$ & & \\
\hline E &
\end{tabular}

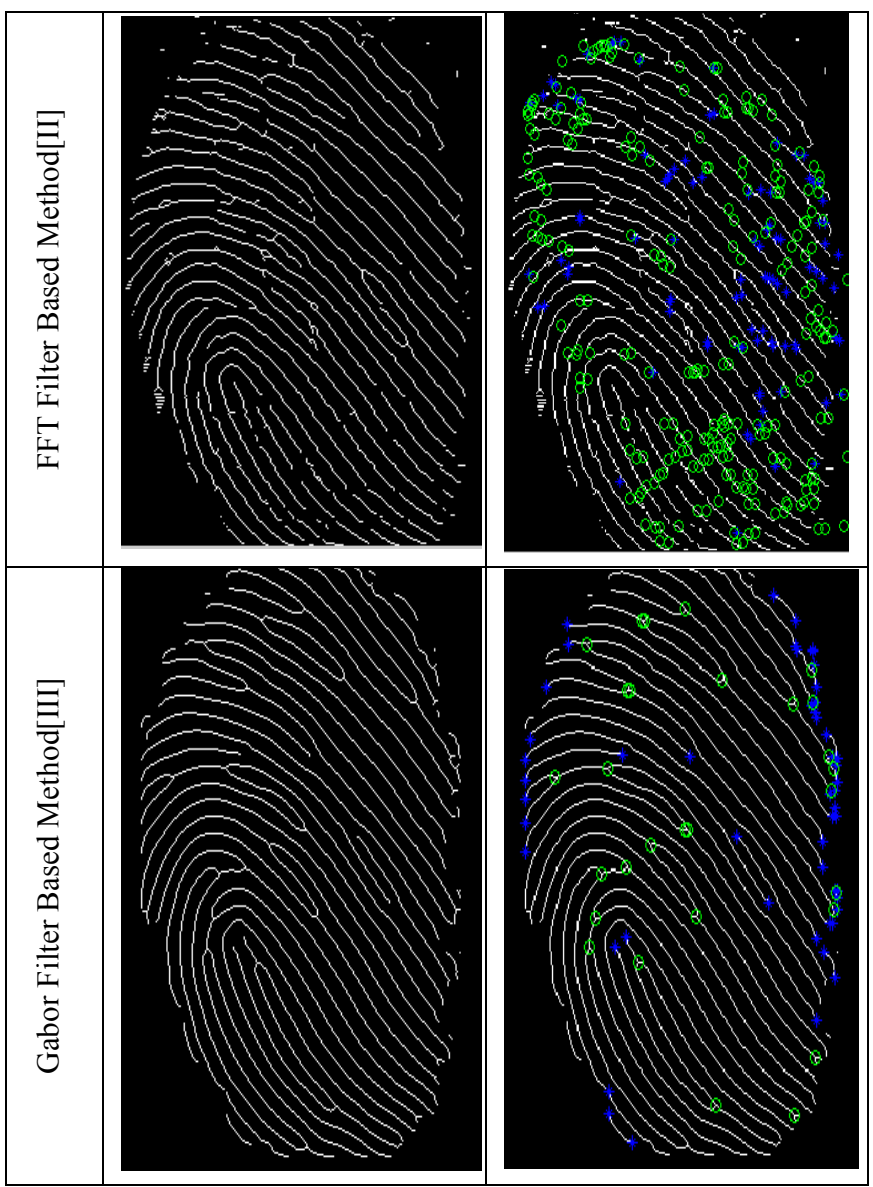

Figure 7: The Output of Three Methods

The following table shows impact of the three image enhancement methods on detection of ridge endings.

Table 1: Detection of Ridge Endings

\begin{tabular}{|c|c|c|c|c|c|}
\hline \multirow{3}{*}{$\begin{array}{l}\dot{z} \\
\dot{\pi}\end{array}$} & \multirow{3}{*}{$\begin{array}{l}\text { Fingerprint } \\
\text { Image }\end{array}$} & \multicolumn{4}{|c|}{$\begin{array}{l}\text { Approximate Count of } \\
\text { Ridge Endings }\end{array}$} \\
\hline & & \multirow{2}{*}{$\begin{array}{c}\text { By } \\
\text { Human } \\
\text { Expert }\end{array}$} & \multicolumn{3}{|c|}{ By Algorithm } \\
\hline & & & I & II & III \\
\hline 1 & 101_1.tif & 20 & 278 & 131 & 52 \\
\hline 2 & 101_2.tif & 15 & 115 & 117 & 19 \\
\hline 3 & 101_3.tif & 17 & 278 & 152 & 31 \\
\hline 4 & 102_1.tif & 20 & 156 & 240 & 65 \\
\hline 5 & 102_2.tif & 21 & 128 & 153 & 48 \\
\hline 6 & 102_3.tif & 20 & 123 & 241 & 54 \\
\hline & Average & 19 & 180 & 172 & 45 \\
\hline \multicolumn{3}{|c|}{$\begin{array}{l}\text { False detection of Ridge } \\
\text { Endings }\end{array}$} & 161 & 153 & 26 \\
\hline
\end{tabular}


From Table 1, its being observed that the algorithm III (Gabor Filter) has detected very minimal false ridge endings than the other two algorithms (Histogram and FFT). Finally the algorithm III is giving an average value of 45 , whereas the other two algorithms 180 and 172 respectively.

The following table shows impact of the three image enhancement methods on detection of ridge Bifurcation.

Table 2: Detection of Ridge Bifurcation

\begin{tabular}{|c|c|c|c|c|c|}
\hline \multirow{3}{*}{$\begin{array}{l}\stackrel{i}{z} \\
\frac{\dot{n}}{n}\end{array}$} & \multirow{3}{*}{$\begin{array}{l}\text { Fingerprint } \\
\text { Image }\end{array}$} & \multicolumn{4}{|c|}{$\begin{array}{l}\text { Approximate Count of } \\
\text { Bifurcation }\end{array}$} \\
\hline & & \multirow{2}{*}{$\begin{array}{c}\text { By } \\
\text { Human } \\
\text { Expert }\end{array}$} & \multicolumn{3}{|c|}{ By Algorithm } \\
\hline & & & I & II & III \\
\hline 1 & 101_1.tif & 16 & 218 & 35 & 29 \\
\hline 2 & 101_2.tif & 14 & 94 & 36 & 17 \\
\hline 3 & 101_3.tif & 17 & 276 & 67 & 25 \\
\hline 4 & 102_1.tif & 26 & 209 & 76 & 67 \\
\hline 5 & 102_2.tif & 24 & 142 & 85 & 42 \\
\hline 6 & 102_3.tif & 17 & 298 & 57 & 23 \\
\hline & Average & 19 & 206 & 59 & 34 \\
\hline \multicolumn{3}{|c|}{$\begin{array}{l}\text { False detection of } \\
\text { Bifurcation }\end{array}$} & 187 & 40 & 15 \\
\hline
\end{tabular}

From Table 2, its being observed that the algorithm III (Gabor Filter) has deducted very minimal false ridge bifurcation than the other two algorithms (Histogram and FFT). Finally the algorithm III is giving an average value of 34, whereas the other two algorithms 206 and 59 respectively.

The following chart compares the average false detection count of ridge endings after three types of image enhancement methods.

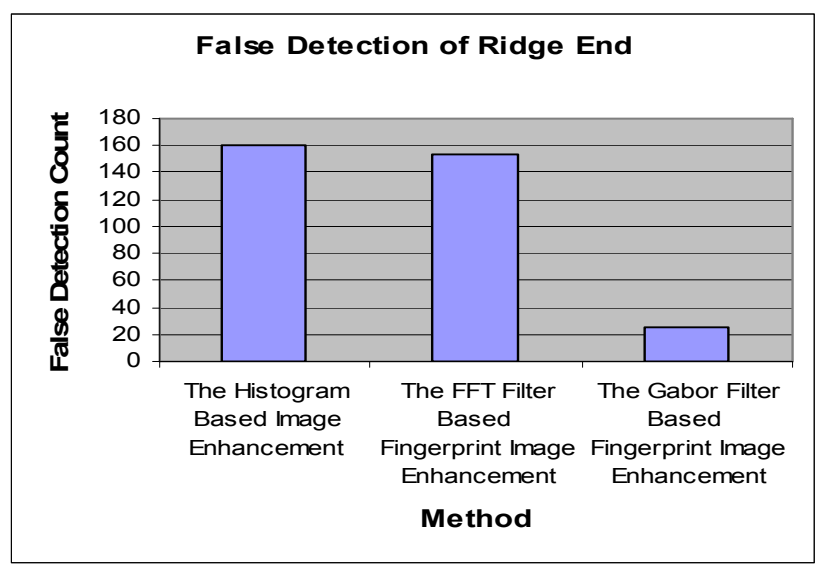

Figure 8: The Output of Three Methods
From figure 8 (constructed form Table 1), we can observe that the average number of false detection of ridge endings is very minimal for Gabor Filter, whereas it's more for the other two methods. This clearly shows that Gabor Filter is the best method for the detection of false ridge endings.

The following chart compares the average false detection count of ridge Bifurcation after three types of image enhancement methods.

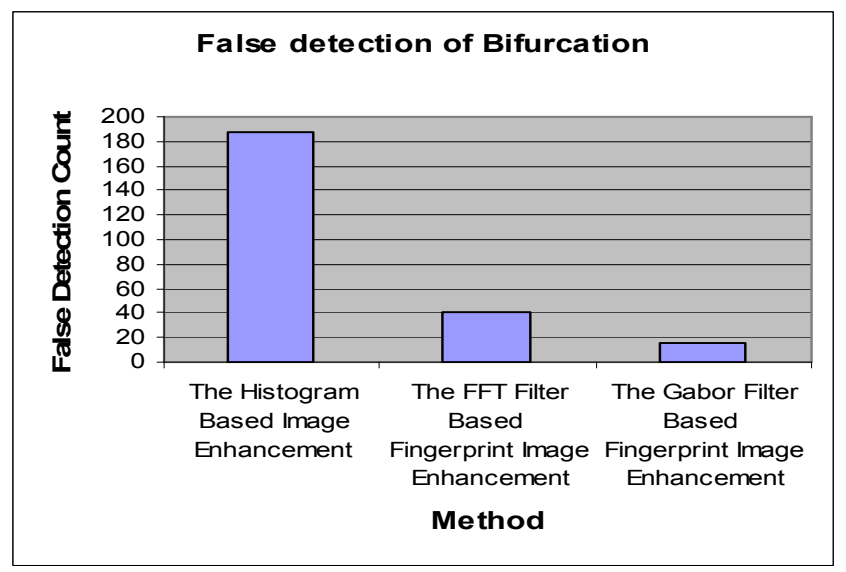

Figure 9: The Output of Three Methods

From figure 9 (constructed form Table 2), we can observe that the average number of false detection of ridge bifurcation is very minimal for Gabor Filter, whereas it's more for the other two methods. This clearly shows that Gabor Filter is the best method for the detection of false ridge endings.

\section{CONCLUSION AND FUTURE WORK}

The Experiment is being successfully implemented and evaluated using three different models under Matlab 6.5. The arrived results were significant and more comparable. It proves the fact that the fingerprint image enhancement step will certainly improves the verification performance of the fingerprint based recognition system. Among the three evaluated fingerprint image enhancement algorithms, the Gabor Filter based algorithm performed very well. It is performed 5 to 6 times better than the other two algorithms. The algorithm enhanced the ridges and valleys in corrupted areas of the fingerprint and this makes the Ridge Ends and Ridge Bifurcation more visible and distinguishable from the image. The better image enhancement algorithm helps in removing the spurious minutia too which may also prove to be harmful in matching fingerprints correctly.

In this work, counting of spurious minutia's alone is considered. In future works, one may address the impact of image enhancement algorithm with the spurious minutia removal algorithms and matching algorithms.

\section{ACKNOWLEDGMENT}

The authors would like to thank the editor and the anonymous reviewers for their valuable comments and suggestions. 


\section{REFERENCES}

[1] Jain A, Hong L, Pankati S and Bolle R. September 1997. An identity authentication system using fingerprints. In Proceedings of the IEEE, vol. 85, pp. 1365-1388.

[2] Maio D and Maltoni D, 1997. Direct gray scale minutia detection in fingerprints, Transactions on PAMI, 19(1).

[3] Maltoni D et al. 2009. Handbook of Fingerprint Recognition, Springer - Verlag London Limited.

[4] Meltemp Ballan and Ayhan Sakarya F and Brian L Evans, 1997. A Fingerprint Classification Technique Using Directional Images. Mathematical and Computational Applications. IEEE, pp. 91-97.

[5] Ranade A and Rosenfeld A, 1993. Point pattern matching by relaxation, Pattern Recognition, 21(2): 269-275.

[6] Ratha N, Chen S and Jain A, 1995. An Adaptive flow orientation based feature extraction in fingerprint images, Pattern Recognition, 1657-1672.
[7] Sherlock, D. B. G, Monro, D. M and Millard K, 1994. Fingerprint enhancement by directional Fourier filtering. In IEEE Proc. Vis. Image Signal Processing, vol. 141, pp. 8794.

[8] Vijay Cornelius John, Fingerprint Based Authentication System, Correlation Based Classification Approach, MSITRT, Robotics Institute, Carnegie Mellon University, Pittsburgh, PA, USA.

[9] WUZHILI, April 2002. Fingerprint recognition, Student project, Hong Kong Baptist University.

[10] Xuejun Tan Bhanu, B. Yingqiang Lin, Aug 2005. Fingerprint classification based on learned features, Center for Res. in Intelligent Syst., Univ. of California, Riverside, CA, USA. 